\title{
Anharmonic Coupling of Stretching Vibrations in Ice: A Periodic VSCF and VCI Description
}

Raymond G. Schireman, ${ }^{\dagger}$ Jefferson Maul, ${ }^{\ddagger}$ Alessandro Erba, ${ }^{*}, \ddagger$ and Michael T. Ruggiero*, $\uparrow$

$\dagger$ Department of Chemistry, University of Vermont, 82 University Place, Burlington, Vermont 05405, United States of America

$\ddagger$ Dipartimento di Chimica, Università di Torino, via Giuria 5, 10125 Torino, Italy

E-mail: alessandro.erba@unito.it; michael.ruggiero@uvm.edu 


\section{Abstract}

The anharmonicity of $\mathrm{O}-\mathrm{H}$ stretching vibrations of water ice is characterized by use of a periodic implementation of the vibrational self-consistent field (VSCF) and vibrational configuration interaction (VCI) methods, which take phonon-phonon couplings explicitly into account through numerical evaluation of high-order terms of the nuclear potential. The lowtemperature, proton-ordered phase of water ice (namely, ice-XI) is investigated. The net effect of a coupled anharmonic treatment of stretching modes is not just a rigid blue-shift of the respective harmonic spectral frequencies but rather a complex change of their relative spectral positions, which can not be captured by simple scaling strategies based on harmonic calculations. The adopted techniques allow for a hierarchical treatment of anharmonic terms of the nuclear potential, which is key to an effective identification of leading factors. We show that an anharmonic independent-mode approximation only describing the "intrinsic anharmonicity" of the $\mathrm{O}-\mathrm{H}$ stretches is unable to capture the correct physics and that couplings among O-H stretches must be described. Inspection of harmonic normal coordinates allows to identify specific features of the O-H stretching motions which most likely enable strong mode-mode couplings. Finally, by coupling O-H stretches to all other possible modes of ice-XI (THz collective vibrations, molecular librations, bendings), we identify specific types of motion which significantly affect O-H stretching states: in particular, molecular librations are found to affect the stretching states more than molecular bendings.

\section{Introduction}

Vibrational dynamics represent one of the most important phenomena in the chemical sciences. Vibrational spectroscopy has been used since the early $20^{\text {th }}$ century as a tool for characterizing interatomic forces and for the identification of molecular species. ${ }^{1}$ Additionally, vibrational states are central to thermodynamic properties (especially for crystalline solids) ranging from entropy and specific heat to thermal expansion. ${ }^{2,3}$ Thus, a complete 
description of vibrational dynamics at the atomic scale is important to fully-understand the bulk properties of solid materials. There are many well-known experimental methods that probe vibrational transitions, such as infrared (IR) and Raman spectroscopies. In addition, many computational chemistry codes can simulate molecular vibrations within the harmonic approximation (HA). The HA, which models vibrational potentials as a quadratic function, is extensively used due to the ability to perform straightforward and computationally efficient analyses. However, despite its popularity, the HA breaks down in situations where the interatomic potential is far from behaving quadratically and/or when different vibrational modes are strongly coupled. The drawbacks of the HA are even more pronounced for solids where, at the harmonic level, there is no volume dependence of thermodynamic properties (i.e., zero thermal expansion), and infinite phonon lifetimes and lattice thermal conductivity. Recently, some of these challenges have been addressed through the use of quasi-harmonic approximation (QHA) simulations, which involve the determination of harmonic vibrational dynamics at a series of crystalline unit cell volumes, providing a holistic description of intermolecular anharmonicity. ${ }^{4-12}$ However, the QHA does not treat vibrational anharmonicity on a mode-by-mode basis, as it still relies on the HA and neglects higher-order (third and above) terms in the expansion of the vibrational potential energy surface (PES), ultimately resulting in the inability to model anharmonic phenomena such as phonon-phonon couplings. ${ }^{13,14}$ While there have been a number of methods proposed for the evaluation of cubic force constants for application to thermal conductivity, ${ }^{15-20}$ as well as the role of mode coupling on anharmonic vibrational states, ${ }^{21-30}$ there is a crucial need for more comprehensive studies of these effects in molecular crystals due to the importance of anharmonic phenomena on bulk material properties.

The focus of this study is the application of the fully-periodic VSCF and VCI methods implemented in CRYSTAL ${ }^{31-35}$ to the O-H stretching vibrations of the low-temperature, proton-ordered phase of water ice (ice-XI) to obtain a description of the anharmonicity due to vibrational mode coupling. Water ice is a molecular crystal of interest for anharmonic analy- 
ses due to its complex network of intermolecular hydrogen bonds coupled with intramolecular covalent $\mathrm{O}-\mathrm{H}$ bonds that complicate the interpretation of the vibrational spectrum and modeling of thermodynamic properties. ${ }^{36-44}$ First, the intrinsic anharmonicity is evaluated and its limits are discussed. Then, mode pair couplings and mode subset couplings are evaluated and their respective advantages are presented. Finally, three factors that can qualitatively predict the degree of coupling in molecular crystals are suggested.

\section{Methods}

All simulations were performed with a developmental version of CRYSTAL17. ${ }^{31}$ Initially, the structure of ice-XI was fully-optimized (i.e. in terms of both lattice vectors and atomic positions) without any constraints other than those imposed by the space group symmetry of the lattice $\left(\mathrm{Cmc} 2_{1}\right)$ with the B3LYP ${ }^{45,46}$ exchange-correlation hybrid functional of the density functional theory (DFT) and a $6-311 \mathrm{G}(2 \mathrm{~d}, 2 \mathrm{p})^{47,48}$ basis set. A tolerance on the SCF convergence of $\Delta E \leq 10^{-10}$ Hartree was used. After optimization, harmonic vibrational analyses were performed on the fully-relaxed geometries of ice-XI using the wellestablished method involving numerical differentiation of analytical forces, with a two-sided finite difference formula to better capture the curvature of the potential energy surface (PES). ${ }^{49,50}$ Infrared (IR) and Raman intensities were calculated analytically through the coupled-perturbed/Kohn-Sham approach. ${ }^{51,52}$

Anharmonic vibrational analyses were performed using a recently reported approach implemented into a developmental version of CRYSTAL17, ${ }^{32,33}$ where the PES is expressed in the basis of mass-weighted normal coordinates, $V(\mathbf{Q})$, and expanded in a Taylor series up 
to fourth order:

$$
\begin{aligned}
V(\mathbf{Q}) & =\frac{1}{2} \sum_{i=1}^{M} \omega_{i}^{2} Q_{i}^{2}+\frac{1}{3 !} \sum_{i, j, k=1}^{M} \eta_{i j k} Q_{i} Q_{j} Q_{k}+ \\
& +\frac{1}{4 !} \sum_{i, j, k, l=1}^{M} \eta_{i j k l} Q_{i} Q_{j} Q_{k} Q_{l}
\end{aligned}
$$

where $M$ is the number of independent quantum harmonic oscillators vibrating at harmonic frequencies $\omega_{i}$ (with $\left.i=1, \ldots, M\right)$ and where $\eta_{i j k}$ and $\eta_{i j k l}$ are cubic and quartic force constants, respectively:

$$
\begin{array}{r}
\eta_{i j k}=\left(\frac{\partial^{3} E}{\partial Q_{i} \partial Q_{j} \partial Q_{k}}\right) \\
\text { and } \eta_{i j k l}=\left(\frac{\partial^{4} E}{\partial Q_{i} \partial Q_{j} \partial Q_{k} \partial Q_{l}}\right)
\end{array}
$$

All one- and two-mode terms in the PES expansion above were computed according to a so-called 2M4T representation of the PES:

$$
\begin{gathered}
\eta_{i i i}, \eta_{i i i i} \quad \forall \quad i \in M \\
\eta_{i j j}, \eta_{i i j}, \eta_{i i i j}, \eta_{i j j j}, \eta_{i i j j} \quad \forall \quad i<j \in M .
\end{gathered}
$$

An Energy-Gradient-Hessian (EGH) two-point finite difference scheme was used to compute such high-order energy derivatives, with a displacement step of 0.9 in terms of classical amplitude of each normal mode. ${ }^{33}$

From such anharmonic potential, anharmonic vibrational states were computed by solving the vibrational self-consistent field (VSCF) equations (where each mode interacts with the average potential of all other modes), and the vibrational configuration interaction (VCI) equations, which allow for a more explicit treatment of mode-mode couplings. Vibrational modes are distinguishable so that the $M$-mode wavefunction of a given vibrational configuration $\mathbf{n}$ does not need to be antisymmetrized and can be written as a Hartree product of 
one-mode functions (modals): ${ }^{32}$

$$
\Phi^{\mathbf{n}}\left(Q_{1}, Q_{2}, \cdots, Q_{M}\right) \equiv \Phi^{\mathbf{n}}(\mathbf{Q})=\prod_{i=1}^{M} \phi_{i}^{n_{i}}\left(Q_{i}\right)
$$

where $\mathbf{n}=\left(n_{1}, n_{2}, \cdots, n_{i}, \cdots, n_{M}\right)$ is the vibrational configuration vector of the quantum numbers of the $M$ one-mode functions. For each given vibrational configuration $\mathbf{n}$, the VSCF method consists in looking for the variationally best form of the corresponding $M$ one-mode functions. This is achieved by requiring that the expectation value of the full Hamiltonian is stationary:

$$
E^{\mathbf{n}}=\left\langle\Phi^{\mathbf{n}}|\mathcal{H}| \Phi^{\mathbf{n}}\right\rangle \quad \text { where } \quad \mathcal{H}=\sum_{i=1}^{M} T_{i}+V(\mathbf{Q})
$$

with $T_{i}=-1 / 2\left(\partial^{2} / \partial Q_{i}^{2}\right)$ being the one-mode kinetic energy operator. In the VCI method, the wave-function of each vibrational state $s$ is written as a linear combination of $M$-mode wave-functions of different vibrational configurations in the form of Hartree products of modals as in Eq. (5):

$$
\Psi_{s}(\mathbf{Q})=\sum_{n=1}^{N_{\text {conf }}} A_{n, s} \Phi^{\mathbf{n}}(\mathbf{Q})
$$

where the sum runs over $N_{\text {conf }}$ configurations, each characterized by a vibrational configuration vector $\mathbf{n}$. The selection of the $N_{\text {conf }}$ configurations determines the truncation of the VCI expansion. For each vibrational state $s$, the corresponding VCI wave-function and energy are obtained by solving the corresponding Schrödinger equation $\mathcal{H} \Psi_{s}=E_{s} \Psi_{s}$. The VCI method can be expressed in matrix form as follows: ${ }^{32} \mathbf{H A}=\mathbf{A E}$, where $\mathbf{A}$ is the squared matrix containing, column-wise, the coefficients $A_{n, s}$ of the eigenvectors, $\mathbf{E}$ is the diagonal matrix of the eigenvalues and $\mathbf{H}$ is the VCI Hamiltonian matrix (of size $N_{\text {conf }} \times N_{\text {conf }}$ ). The VCI method therefore reduces to the construction and diagonalization of the VCI Hamiltonian matrix, from which all vibrational states are simultaneously determined. VSCF solutions are used to express the modals in the VCI method (according to the so-called VCI@VSCF approach). 
The limiting factor of the VCI method is the construction of the VCI Hamiltonian matrix, which can easily exceed the memory capacity of most nodes. Erba et al. developed several strategies for truncating the VCI expansion and thus the size of the Hamiltonian matrix by exploiting point-symmetry factorization and by limiting the number of quanta and modes simultaneously excited. ${ }^{32}$ For fundamental transition frequencies of ice-XI, Erba et al. ${ }^{32}$ determined that the VCI@VSCF method converges when all configurations involving up to four quanta over up to three different modes are considered, which is the set-up used here.

When the anharmonicity of a single mode is considered (i.e. by neglecting all couplings with other modes), we refer to the intrinsic anharmonicity of the mode. In order to rationalize the impact that different mode-mode couplings have on the description of anharmonic stretching states, we have grouped ice-XI modes into four subsets by their spectral region and mode type: terahertz, librations, bends, and O-H stretches. Such classification allows for a hierarchical treatment of mode-mode couplings to identify the most relevant ones.

\section{Results and Discussion}

\section{Structural Analysis}

Before lattice dynamics can be investigated, it is important to ensure that the geometry optimization converged to a structure that is at an equilibrium point on the PES, as well as agreeing with the experimental structure, which helps to ensure that the interatomic and intermolecular forces are well-modeled. ${ }^{53,54}$ The structure of ice-XI was previously determined experimentally using neutron diffraction at $10 \mathrm{~K} .{ }^{55}$ Ice-XI crystallizes in the $C m c 2_{1}$ orthorhombic space group with lattice parameters of $4.5026 \AA, 7.7803 \AA, 7.2884 \AA$ for $a, b$, and $c$ respectively. ${ }^{55}$ The B3LYP/6-311G(2d,2p) optimization successfully reproduced the

experimental structure, with lattice parameters of $4.4859 \AA, 7.7623 \AA$, and $7.3194 \AA$, representing an average relative error in the lattice vectors of $-0.059 \%$. 


\section{Harmonic Analysis}

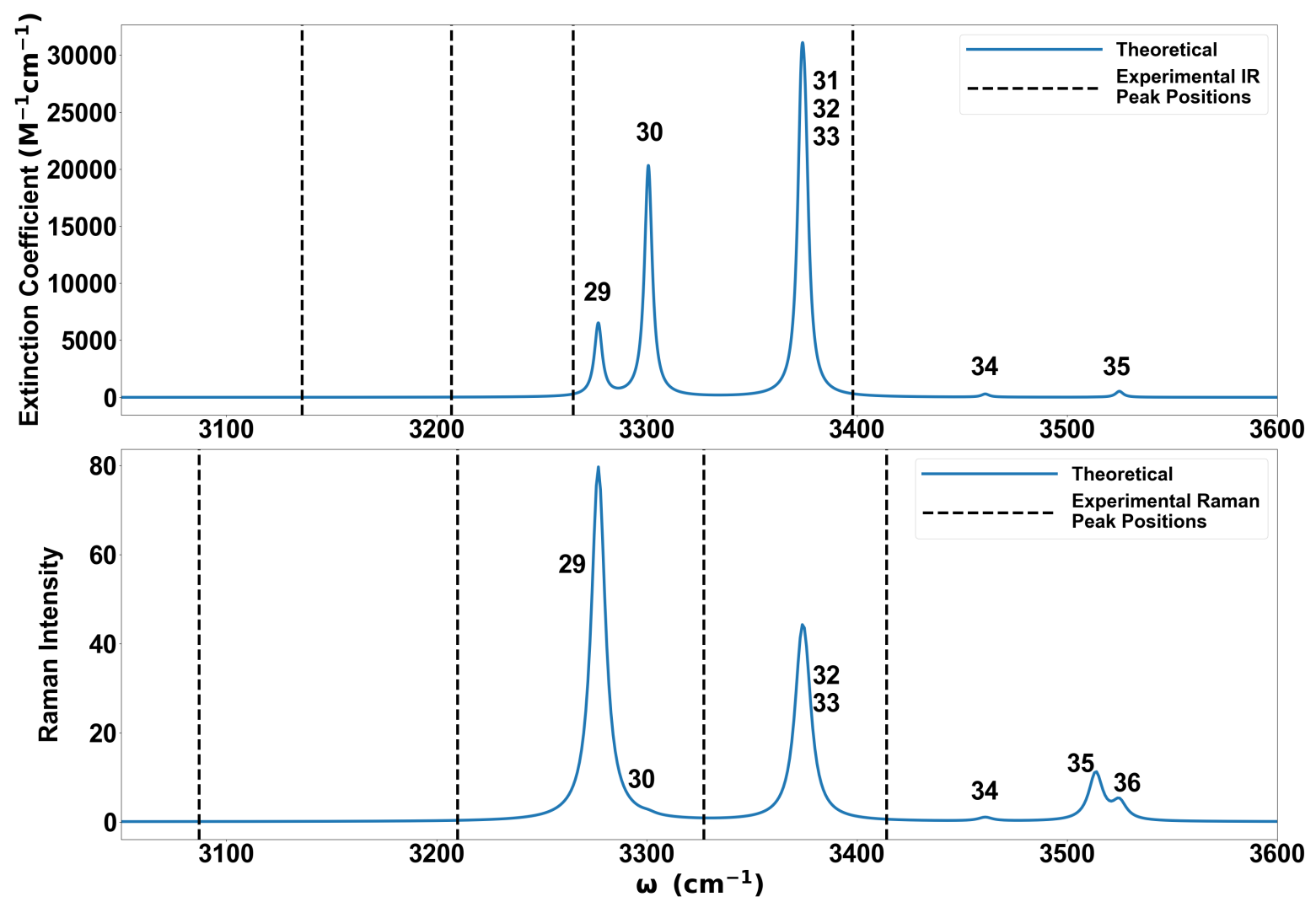

Figure 1: Simulated harmonic vibrational spectra of ice-XI in the O-H stretching region (IR in the upper panel and Raman in the lower panel). Dashed vertical lines mark the positions of the experimental peaks. ${ }^{56}$ Assignments of each peak in the spectra to the individual normal modes are provided by the numerical labels.

Following the successful optimization, harmonic vibrational analysis was performed. The calculated IR and Raman spectra of ice-XI in the O-H stretching region are shown in Figure 1, with black vertical dashed lines marking the positions of the experimental peaks. Indeed, we will primarily examine the $\mathrm{O}-\mathrm{H}$ stretching modes in-depth as they have been extensively characterized both experimentally and theoretically. However, we have included all simulation data in the ESI so as to make these rich datasets available. The eight stretching modes of ice-XI were previously assigned based on symmetry by Erba et al. as follows: ${ }^{57}$

$$
3 A_{1}+3 B_{1}+A_{2}+B_{2}
$$


Based on the theoretical analysis, almost all 33 optical modes of ice-XI are both IR and Raman active, with the exception being mode 31, which is only IR inactive. The predicted harmonic frequencies of modes 31, 32 and 33 are incredibly similar $\left(3373,3374\right.$ and $3375 \mathrm{~cm}^{-1}$, see Table 1), thus these modes would almost certainly be indistinguishable in experimental studies. However, we are going to show later how these three modes are split when modemode couplings are taken into account beyond the independent mode approximation. It is important to note that there are eight theoretical stretching modes (with assignments given in Eq. 8), four bands observed in experimental IR spectra, and four bands in experimental Raman spectra of ice-XI. Indeed, Shigenari and Abe performed an experimental analysis of the vibrational modes above $400 \mathrm{~cm}^{-1}$ of ice-XI via IR and Raman measurements. ${ }^{56}$ In the Raman spectrum of ice-XI, Shigenari and Abe observed four peaks at $3087 \mathrm{~cm}^{-1}, 3210$ $\mathrm{cm}^{-1}, 3327 \mathrm{~cm}^{-1}$, and $3414 \mathrm{~cm}^{-1} .{ }^{56}$ Four peaks were also observed in the IR spectrum at 3136 $\mathrm{cm}^{-1}, 3207 \mathrm{~cm}^{-1}, 3265 \mathrm{~cm}^{-1}$, and $3398 \mathrm{~cm}^{-1} .{ }^{56}$ Based on the purely harmonic description, a tentative assignment can be attempted: in the Raman spectrum, the peak at $3087 \mathrm{~cm}^{-1}$ would correspond to mode 29 (an in-phase symmetric stretching on all four water molecules; see Figure 2), that at $3210 \mathrm{~cm}^{-1}$ to modes 32 and 33, that at $3327 \mathrm{~cm}^{-1}$ to mode 34 and the peak at $3414 \mathrm{~cm}^{-1}$ to modes 35 and 36, which have similar harmonic frequencies and Raman intensities of 135.53 a.u. and 51.07 a.u., respectively. In the IR spectrum, the peak at 3136 $\mathrm{cm}^{-1}$ would correspond to mode 30 , that at $3207 \mathrm{~cm}^{-1}$ to modes 31,32 and 33 , that at 3265 $\mathrm{cm}^{-1}$ to mode 34 and the peak at $3398 \mathrm{~cm}^{-1}$ to modes 35 and 36 , which have similar harmonic frequencies and IR intensities of $2.37 \times 10^{5} \mathrm{M}^{-1} \mathrm{~cm}^{-1}$ and $1.88 \times 10^{6} \mathrm{M}^{-1} \mathrm{~cm}^{-1}$, respectively.

From the analysis above based on Figure 1, the frequencies calculated by the harmonic approximation only allow for a tentative assignment of experimental peaks as they do not just differ quantitatively from experimental ones in terms of absolute values (with an average blue-shift of about $147 \mathrm{~cm}^{-1}$ ) but also qualitatively in terms of relative positions. We are going to show and discuss below how an explicit anharmonic treatment taking into account mode-mode couplings is required to get improved relative positions and assignment. 

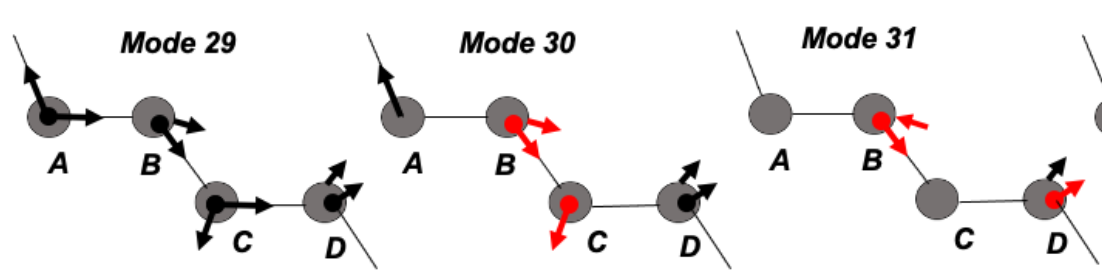

Mode 32
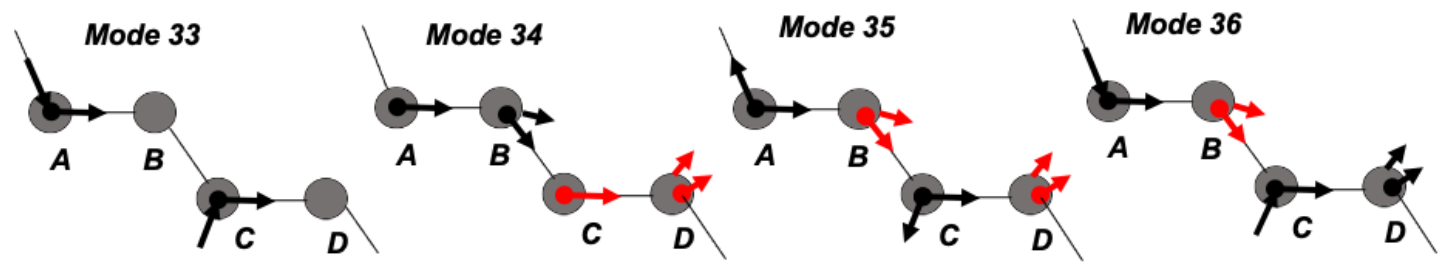

Figure 2: Eigenvectors obtained from the normal mode analysis. The eigenvectors of each mode are indicated by black arrows on the four water molecules in the unit cell of ice-XI. The water molecules are labeled by capital letters (A,B,C, and D). The relative phase is indicated by the color of the arrows.

In order to guide the interpretation of the tentative harmonic assignment just presented as well as the mode-mode couplings that will follow, Figure $\mathbf{2}$ presents a skematic graphical representation of the harmonic normal mode eigenvectors of the eight O-H stretching modes in ice XI. The four water molecules in the lattice cell are labeled A, B, C and D. Stretching motions are represented by arrows and phase of motion is indicated by color. For example, the symmetric stretch on water molecule B in mode 30 is out of phase with the symmetric stretch on water molecules A and D. Also, the symmetric stretch on water molecule $\mathrm{C}$ is out of phase with the symmetric stretch on water molecules A and D, but is in phase with the symmetric stretch on water molecule B since they both have the same color for the displacement arrows.

\section{Intrinsic Anharmonicity}

The first anharmonic treatment of $\mathrm{O}-\mathrm{H}$ stretching vibrations in ice-XI we performed was a single-mode one, where the intrinsic anharmonicity of each normal mode is investigated by accounting for cubic and quartic single-mode terms in the PES (i.e. terms $\eta_{i i i}$ and $\eta_{i i i i}$ in Eq. 1) and by neglecting mode-mode couplings. In other words, normal modes were still considered as independent but the non-quadraticity of their 1D potential was accounted for. 
In this limit, the VSCF and VCI methodologies formally coincide. The obtained results, in terms of intrinsic anharmonic frequencies of fundamental transitions $\left(\omega_{i}^{\mathrm{ia}}\right)$ are reported in Table 1.

Table 1: Fundamental O-H stretching frequencies of ice-XI as computed with the harmonic approximation and by taking into account just the intrinsic anharmonicity of each vibrational mode. Labels of symmetry irreps of each mode are also listed. The last column, $\Delta$, represents the difference between intrinsic anharmonic and harmonic values. Values in $\mathrm{cm}^{-1}$.

\begin{tabular}{ccccc} 
Mode \# & $\begin{array}{c}\text { Symmetry } \\
\text { Label }\end{array}$ & Harmonic & $\begin{array}{c}\text { Intrinsic } \\
\text { Anharmonicity }\end{array}$ & $\boldsymbol{\Delta}$ \\
\hline \hline $\mathbf{2 9}$ & A1 & 3277 & 3225 & -52 \\
$\mathbf{3 0}$ & B1 & 3302 & 3338 & +36 \\
$\mathbf{3 1}$ & A2 & 3373 & 3422 & +59 \\
$\mathbf{3 2}$ & A1 & 3374 & 3422 & +58 \\
$\mathbf{3 3}$ & B2 & 3375 & 3422 & +57 \\
$\mathbf{3 4}$ & B1 & 3461 & 3514 & +53 \\
$\mathbf{3 5}$ & A1 & 3514 & 3533 & +19 \\
$\mathbf{3 6}$ & B1 & 3525 & 3548 & +23 \\
\hline \hline
\end{tabular}

From inspection of the data reported in the table, the following considerations can be made: i) the intrinsic anharmonicity produces an increase of the fundamental vibration frequency of all stretching modes but the lowest frequency one (namely mode 29: the symmetric in-phase stretching on all four water molecules in the cell); ii) the absolute shift in frequency is quite large (up to between $50-60 \mathrm{~cm}^{-1}$ for modes $31-34$, for instance); iii) apart from mode 29 , the shift in frequency of all other stretching modes due to the intrinsic anharmonicity is such to worsen the agreement with the experimental values rather than to improve it. While such behavior may seem counterintuitive with respect to what one is used to find in the description of $\mathrm{O}-\mathrm{H}$ stretching modes in a single water molecule (where a simple Morse-like model would predict a lowering of the frequency upon inclusion of high-order terms of the PES), it is fully justified by the compact 3D network of hydrogen-bonds interconnecting water molecules in ice. In other words, while in an isolated water molecule hydrogen atoms move towards dissociation, in ice they move towards the next oxygen atom in their stretching vibration mode. The use of the sole intrinsic anharmonicity can still prove useful in a con- 
densed matter context, particularly so when considering low-frequency, collective vibrations in the terahertz region of the spectrum. ${ }^{58-60}$ However, this is clearly not the case for O-H stretching modes in ice. Therefore, we proceeded by taking into explicit account mode-mode couplings via the VSCF and VCI methods.

\section{Anharmonicity through Mode Pair Couplings}

From the analysis above, it is obvious that an anharmonic independent-mode approximation is unable to catch the correct behavior of $\mathrm{O}-\mathrm{H}$ stretching vibrations in ice. Mode-mode couplings need to be explicitly taken into account. In order to rationalize what couplings are the most relevant to a correct description of the $\mathrm{O}-\mathrm{H}$ stretching motions in ice, we have devised a hierarchical treatment that starts by coupling just two modes at a time, then couples all modes within a certain subset (e.g. librations, bends, stretchings), then all modes belonging to selected subsets, and finally couples all modes at once.

Let us start by investigating the strength of the coupling between single pairs of modes. We have run as many VSCF and VCI calculations as there are pairs of vibration modes in ice XI, $\left(Q_{i}, Q_{j}\right)$ with $i, j=1, \ldots, M$. Each calculation takes into account the third- and fourthorder coupling constants involving just the two respective modes, as in Eq. (4). In order to quantify the effect of the pair coupling on the vibrational states, we analyse a vibrational state where both modes of the pair are simultaneously singly exited (so-called "combination bands" in vibrational spectroscopies). In other words, for each selected pair of modes, we consider the vibrational configuration $\mathbf{n}=\left(n_{1}, n_{2}, \cdots, n_{k}, \cdots, n_{M}\right)$ with $n_{k}=\delta_{k i}+\delta_{k j}$, where $\delta$ is Kronecker's delta function. From the VSCF approach, we get the energy $E^{\mathbf{n}}$ of this state from Eq. (6) so that its transition frequency $\omega_{i j}^{\text {pair }}$ can be obtained from $\hbar \omega_{i j}^{\text {pair }}=E^{\mathbf{n}}-E^{\mathbf{0}}$, where $\mathbf{0}$ is the fundamental state. From the VCI approach, we search for the vibrational state $s^{\prime}$ with the strongest $\mathbf{n}$ character - i.e. with the largest $A_{n, s}$ coefficient in Eq. (7). The corresponding transition frequency can thus be obtained from $\hbar \omega_{i j}^{\text {pair }}=E_{s^{\prime}}-E_{0}$.

In order to measure the effect of the anharmonic pair mode coupling over an independent 
mode anharmonic treatment (as in the intrinsic anharmonic description discussed before), we compare the transition frequency for this two-mode state $\omega_{i j}^{\text {pair }}$ with the sum of the singlyexcited transition frequencies, $\omega_{i}^{\text {ia }}$ and $\omega_{j}^{\text {ia }}$ of the two individual modes treated at the intrinsic anharmonic level. We define the absolute difference (AD) as:

$$
\Delta \omega_{i j}^{\mathrm{AD}}=\omega_{i j}^{\mathrm{pair}}-\left(\omega_{i}^{\mathrm{ia}}+\omega_{j}^{\mathrm{ia}}\right)
$$

In order to account for relatively large (or small) changes in frequency, the percent difference (PD) was calculated relative to the sum of the singly-excited frequencies:

$$
\Delta \omega_{i j}^{\mathrm{PD}}=\frac{\omega_{i j}^{\mathrm{pair}}-\left(\omega_{i}^{\mathrm{ia}}+\omega_{j}^{\mathrm{ia}}\right)}{\left(\omega_{i}^{\mathrm{ia}}+\omega_{j}^{\mathrm{ia}}\right)} \times 100
$$

In either case, $\Delta \omega_{i j}^{\mathrm{AD}}$ and $\Delta \omega_{i j}^{\mathrm{PD}}$ quantify the coupling between a given pair of modes. A large $\Delta \omega_{i j}$ means that the frequency of the doubly excited state cannot be estimated just by summing the two frequencies of the corresponding individual modes. Figure 3 provides a graphical representation of the analysis outlined above. Each panel of the figure shows (in a color scale) the strength of the pair coupling for each pair of normal modes in ice XI $\left(Q_{i}, Q_{j}\right)$ as quantified by Eqs. (9) and (10) from both a VSCF (top) and VCI (bottom) approach.

The plots in Figure 3 can be interpreted as follows. If two modes do not strongly couple, $\Delta \omega_{A D}$ and $\Delta \omega_{P D}$ should be near zero, as this implies the doubly-excited state has the same (or nearly the same) energy as the sum of each mode being singly excited independently from the other. Conversely, when the two modes are strongly coupled, there will be a large deviation between the doubly excited state energy and the energies of the two singly-excited modes. Inspection of Figure 3 allows some considerations: i) the description of the pair coupling strength from the VSCF and VCI methods appears very consistent, with both approaches describing the same qualitative pattern with small differences in the absolute values; ii) weak couplings are observed between stretching and $\mathrm{THz}$ vibrations, as well as between bendings and $\mathrm{THz}$ vibrations (both cases involve mode types with very different 

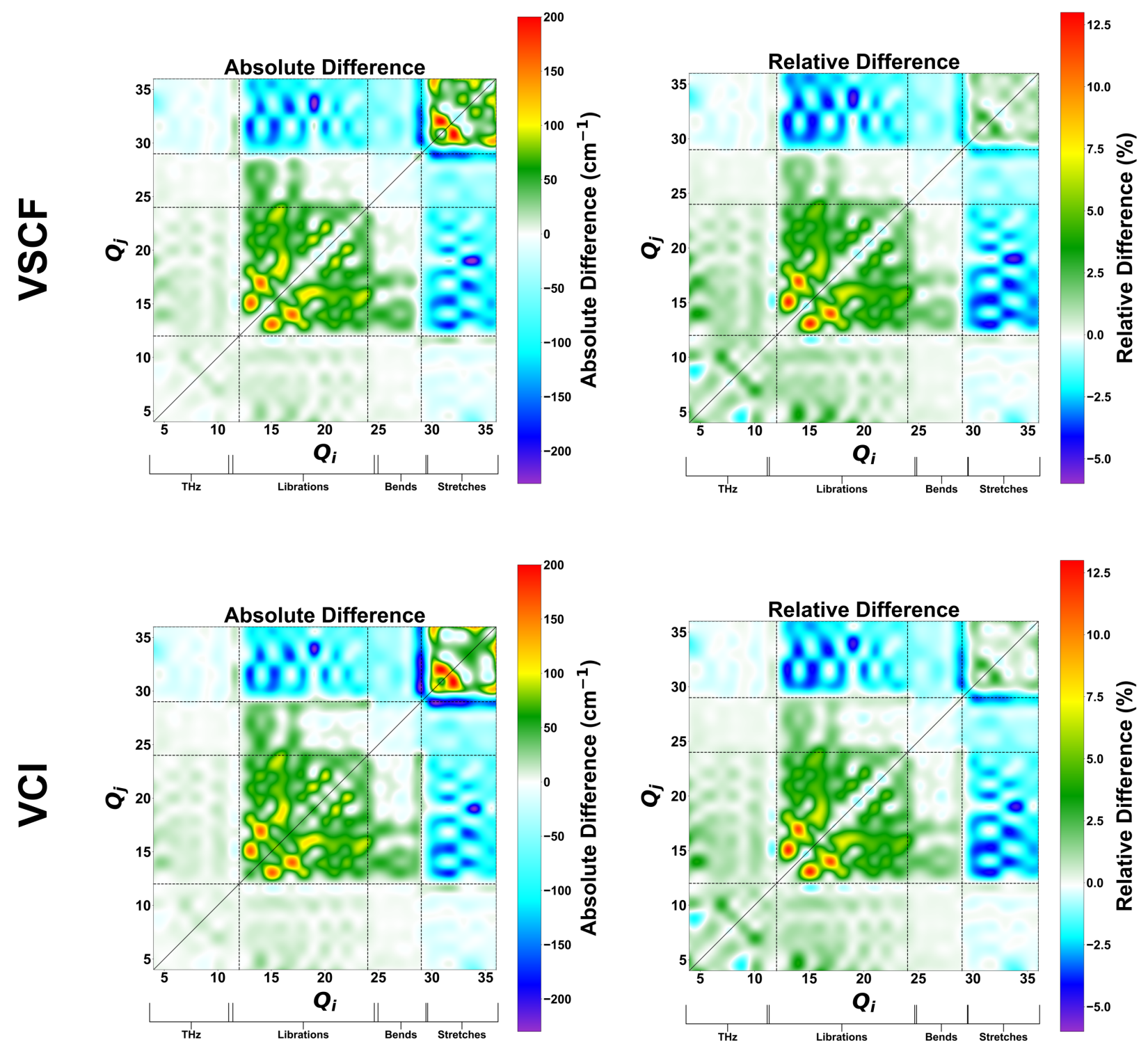

Figure 3: Vibrational two-quanta excited-state pair coupling space of ice XI. For each pair $\left(Q_{i}, Q_{j}\right)$ of normal modes, $\Delta \omega_{i j}^{\mathrm{AD}}$ (left) and $\Delta \omega_{i j}^{\mathrm{PD}}$ (right) are reported, as defined in Eqs. (9) and (10), respectively. Results are shown both from a VSCF (top) and VCI (bottom) approach.

energies); iii) interestingly, weak couplings are observed among bending modes (actually, this is the only diagonal block of the matrix showing very small values); iv) all other diagonal blocks of the matrix show relatively large values, which implies a relatively strong coupling among stretching modes, among librational modes and also among THz modes (this latter case is more evident from the analysis of the $\mathrm{PD}$ rather than the $\mathrm{AD}$, as expected because 
of the low absolute frequencies of THz vibrations); v) Among the stretching diagonal block, mode 29 (i.e. the symmetric in-phase stretching on all four water molecules) once again behaves differently from other modes yielding negative $\Delta \omega_{i j}$ with all other stretching modes at variance with the positive $\Delta \omega_{i j}$ of all other stretching mode pairs; vi) Some off-diagonal blocks also show large values, indicative of relatively strong couplings between modes of different spectral subsets (this is the case of the stretching-bending block and even more so of the libration-stretching, libration-bending and libration-THz, which highlights the key role played by the librational motions in the anharmonic behavior of ice, with librations able to couple with all other vibrations).

\section{Anharmonicity through Couplings among Different Subsets of Modes}

While the two-mode couplings analyzed above have allowed to identify what types of modes are most likely to contribute more strongly to the anharmonic description of vibrational states in ice XI, they still represent an over-simplified description of anharmonicity. Indeed, in principle, all modes of a system could couple with each other. In order to investigate this, we have grouped the 33 modes of ice XI into four subsets ( $\mathrm{THz}$ collective vibrations, librations, bends, and stretches) and progressively allowed the stretches to couple with modes of other subsets by using the VCI approach. The results of this analysis are presented in Figure 4, where the fundamental O-H stretching frequencies of ice XI are reported as a function of the level of coupling in the anharmonic treatment. In particular, harmonic values are reported on the left of the plot, followed by the corresponding "intrinsic anharmonic" values (still obtained from an independent mode approximation), then by values obtained by letting all stretching modes couple with each other, then by letting all stretching modes couple with each other and with all $\mathrm{THz}$ modes, then by letting all stretching modes couple with each other and with all bending modes, then by letting all stretching modes couple with each other and with all libration modes, then by letting all stretching modes couple with each other, with all bending and all libration modes, and finally (on the right of the plot) by 
letting all 33 modes couple with each other.

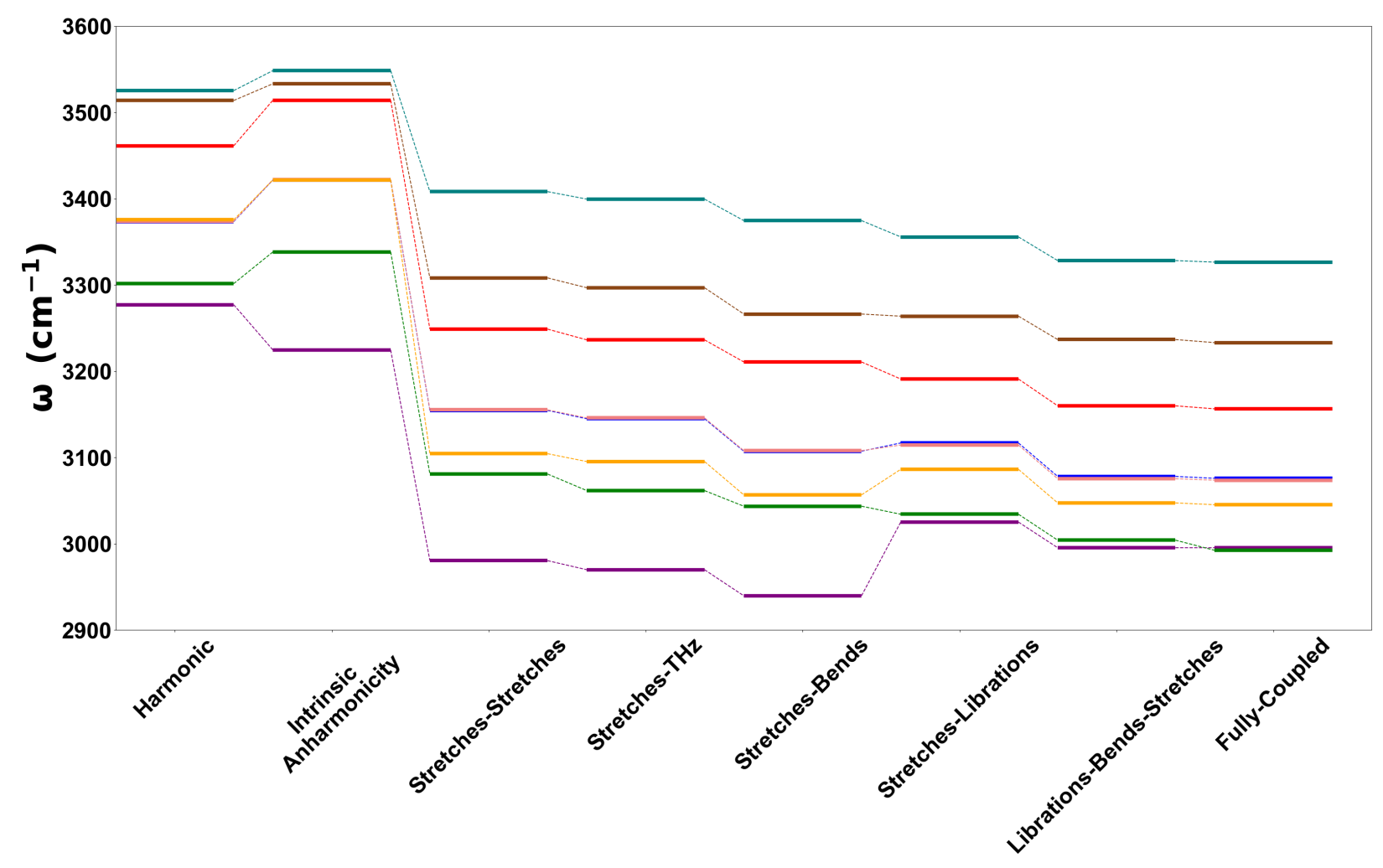

Figure 4: Effect of an incremental VCI treatment of anharmonicity on fundamental O-H stretching frequencies of ice XI. Harmonic values are reported on the left of the plot, followed by the corresponding "intrinsic anharmonic" values (still obtained from an independent mode approximation), then by values obtained by letting all stretching modes couple with each other, then by letting all stretching modes couple with each other and with all $\mathrm{THz}$ modes, and so on up to the right of the plot where values obtained by letting all modes couple with each other are reported.

The effect of the "intrinsic anharmonicity" of the $\mathrm{O}-\mathrm{H}$ stretches has already been discussed and leads to an increase of the frequencies of all modes but the lowest energy one (mode 29, the symmetric in-phase stretching of all four water molecules in the cell), which instead decreases. When stretching modes are allowed to couple with each other we observe three significant effects: i) the pronounced lowering of all O-H stretching frequencies; ii) the relative lowering in energy of mode 32 (brown) with respect to mode 33 (turquoise) while the two are very close in energy in the independent mode approximation; iii) the splitting of the three modes (31, 32 and 33, characterized by a very close harmonic frequency) into a single mode 
with lower frequency (yellow mode in the Figure) and two modes still exhibiting a similar frequency (blue and orange modes in the Figure). If stretching modes are further allowed to couple with $\mathrm{THz}$ vibrations, no significant change in their fundamental transition frequencies are observed. A slightly more pronounced effect is produced by coupling the stretches with the bends, which goes in the direction of further lowering the frequencies of the stretches. As expected from the pair coupling analysis of Figure 3, the coupling of the stretches with the librations produces a significant further red-shift of all O-H stretching frequencies, with the exception - once again - of the symmetric in-phase stretching, whose frequency is blueshifted instead, getting very close in value to the second lowest energy state. The energy difference between these two states is further reduces by the simultaneous coupling of all stretches, bends and librations and eventually vanishes when all 33 modes are coupled with each other, leading to the spectral distribution of the O-H stretches in ice XI shown on the right of Figure 4.

Clearly, couplings among stretching modes are the most critical ones for a correct description of O-H stretching vibrational states in ice. In the Supporting Information, we present a detailed analysis of individual couplings between all pairs of stretches and, based on the analysis of the respective normal modes sketched in Figure 2, we identify some factors, which help predict the strength of anharmonic pair couplings among stretches:

1. Motion on the same molecules of the unit cell in the two modes;

2. Relative phase of the motions in each molecule in the two modes (in-phase stretches on the same molecule in the two modes being optimal);

3. Symmetry of motion (symmetric stretches couple best to asymmetric stretches, and vice-versa).

To better highlight the different spectral distribution of the $\mathrm{O}-\mathrm{H}$ stetching modes when passing from an independent mode approximation to a coupled one, we introduce Figure 5, where simulated frequencies are also compared to experimental ones extracted from peak 


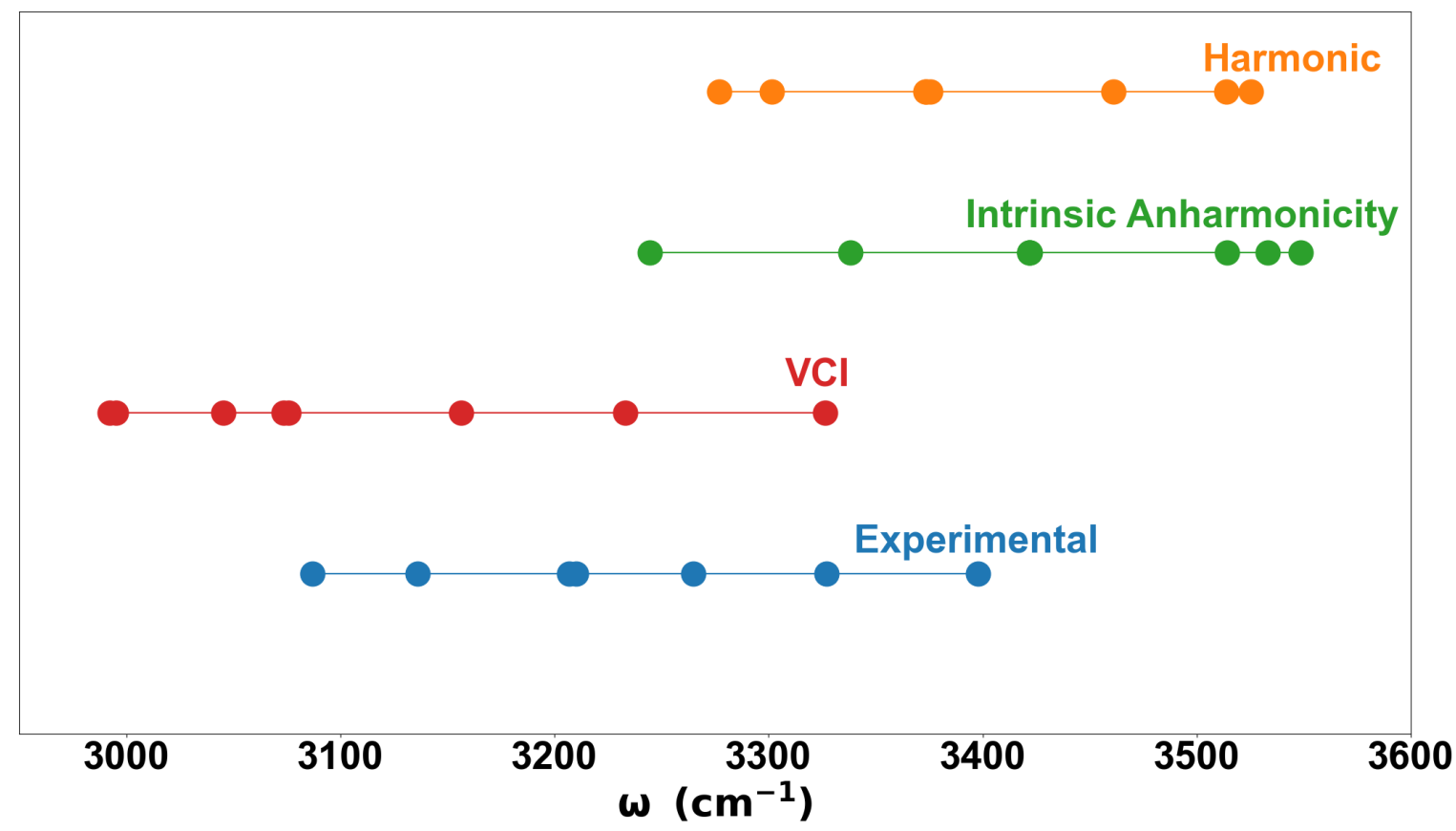

Figure 5: Comparison of the vibrational frequencies of $\mathrm{O}-\mathrm{H}$ stretching modes in ice XI as predicted by the harmonic approximation, by an "intrinsic anharmonicity" independent mode treatment, and by the coupled VCI approach with experimental peak positions from IR and Raman spectra. ${ }^{56}$

positions of IR and Raman spectra. ${ }^{56}$ A first quick look at the Figure reveals the different spectral ranges of the various sets of data, with the harmonic approximation describing the $\mathrm{O}-\mathrm{H}$ stretches as largely blue-shifted with respect to the experiment and spanning a narrower energy range. The energy range widens upon inclusion of intrinsic anharmonic effects and eventually the frequencies are lowered by mode-mode couplings as accounted for by the VCI method. The matching of experimental and VCI energy ranges is not perfect but this aspect has little relevance in this context as this critically depends on the adopted exchange-correlation functional and could be easily tuned by varying the fraction of Fock exchange used in hybrid functionals, for instance. What we find much more relevant here is the evolution of the relative positions of the frequencies (i.e. the pattern of the filled circles in the Figure) when passing from an independent mode approach to a coupled mode approach. Indeed, the three theoretical sets are characterized by very different patterns, with that from the coupled VCI approach closely matching that from the experiments. 
These findings have profound practical implications in computational spectroscopy of strongly anharmonic systems as they clearly show how a simple uniform scaling of harmonic frequencies may result in a correct frequency range but incorrect relative spectral distribution of different spectral features. 


\section{Acknowledgments}

MTR and RS thank the National Science Foundation (award numbers CHE-2055402 and

DMR-2046483), the American Chemical Society Petroleum Research Fund (61794-DNI10), and the University of Vermont for support. 


\section{References}

(1) Gupta, V. In Principles and Applications of Quantum Chemistry; Gupta, V., Ed.; Academic Press: Boston, 2016; pp 247-289.

(2) Born, M.; Huang, K. Dynamical theory of Crystal Lattices; Clarendon Press, 2002.

(3) Hill, T. L. An Introduction to Statistical Thermodynamics; Dover Publications, 2012.

(4) Allen, R. E.; De Wette, F. W. Calculation of Dynamical Surface Properties of NobleGas Crystals. I. The Quasiharmonic Approximation. Phys. Rev. 1969, 179, 873-886.

(5) Baroni, S.; Giannozzi, P.; Isaev, E. Density-Functional Perturbation Theory for QuasiHarmonic Calculations. Rev. Mineral. Geochem. 2010, 71, 39-57.

(6) Erba, A. On Combining Temperature and Pressure Effects on Structural Properties of Crystals with Standard ab initio Techniques. J. Chem. Phys. 2014, 141, 124115.

(7) Erba, A.; Shahrokhi, M.; Moradian, R.; Dovesi, R. On How Differently the Quasiharmonic Approximation Works for Two Isostructural Crystals: Thermal Properties of $\mathrm{MgO}$ and CaO. J. Chem. Phys. 2015, 142, 044114.

(8) Erba, A.; Maul, J.; Demichelis, R.; Dovesi, R. Assessing Thermochemical Properties of Materials through Ab initio Quantum-mechanical Methods: The Case of $\alpha-\mathrm{Al}_{2} \mathrm{O}_{3}$. Phys. Chem. Chem. Phys. 2015, 17, 11670-11677.

(9) Erba, A.; Maul, J.; De La Pierre, M.; Dovesi, R. Structural and Elastic Anisotropy of Crystals at High Pressure and Temperature from Quantum-mechanical Methods: The Case of $\mathrm{Mg}_{2} \mathrm{SiO}_{4}$ Forsterite. J. Chem. Phys. 2015, 142, 204502.

(10) Erba, A.; Maul, J.; Itou, M.; Dovesi, R.; Sakurai, Y. Anharmonic Thermal Oscillations of the Electron Momentum Distribution in Lithium Fluoride. Phys. Rev. Lett. 2015, $115,117402$. 
(11) Erba, A.; Maul, J.; Civalleri, B. Thermal Properties of Molecular Crystals through Dispersion-corrected Quasi-harmonic Ab initio Calculations: The Case of Urea. Chem. Commun. 2016, 52, 1820-1823.

(12) Destefanis, M.; Ravoux, C.; Cossard, A.; Erba, A. Thermo-Elasticity of Materials from Quasi-Harmonic Calculations. Minerals 2019, 9, 16.

(13) Leibfried, G.; Ludwig, W. In Theory of Anharmonic Effects in Crystals; Seitz, F., Turnbull, D., Eds.; Solid State Physics; Academic Press, 1961; Vol. 12; pp 275 - 444.

(14) Plakida, N. M.; Siklós, T. Theory of Anharmonic Crystals. I. General Formulation. Phys. Status Solidi B 33, 103-112.

(15) Togo, A.; Chaput, L.; Tanaka, I. Distributions of Phonon Lifetimes in Brillouin Zones. Phys. Rev. B 2015, 91, 094306.

(16) Togo, A.; Tanaka, I. First Principles Phonon Calculations in Materials Science. Scr. Mater. 2015, 108, $1-5$.

(17) Plata, J. J.; Nath, P.; Usanmaz, D.; Carrete, J.; Toher, C.; de Jong, M.; Asta, M.; Fornari, M.; Nardelli, M. B.; Curtarolo, S. An Efficient and Accurate Framework for Calculating Lattice Thermal Conductivity of Solids: AFLOW-AAPL Automatic non Library. NPJ Comput. Mater. 2017, 3, 45.

(18) Skelton, J. M.; Parker, S. C.; Togo, A.; Tanaka, I.; Walsh, A. Thermal Physics of the Lead Chalcogenides PbS, PbSe, and PbTe from First Principles. Phys. Rev. B 2014, 89, 205203.

(19) Whalley, L. D.; Skelton, J. M.; Frost, J. M.; Walsh, A. Phonon Anharmonicity, Lifetimes, and Thermal Transport in $\mathrm{CH}_{3} \mathrm{NH}_{3} \mathrm{PbI}_{3}$ from Many-body Perturbation Theory. Phys. Rev. B 2016, 94, 220301. 
(20) Linnera, J.; Karttunen, A. J. Ab initio Study of The Lattice Thermal Conductivity of $\mathrm{Cu}_{2} \mathrm{O}$ Using the Generalized Gradient Approximation and Hybrid Density Functional Methods. Phys. Rev. B 2017, 96, 014304.

(21) Werthamer, N. R. Self-Consistent Phonon Formulation of Anharmonic Lattice Dynamics. Phys. Rev. B 1970, 1, 572-581.

(22) Tadano, S., T.and Tsuneyuki Self-consistent Phonon Calculations of Lattice Dynamical Properties in Cubic $\mathrm{SrTiO}_{3}$ with First-principles Anharmonic Force Constants. Phys. Rev. B 2015, 92, 054301.

(23) Tadano, T.; Tsuneyuki, S. Quartic Anharmonicity of Rattlers and Its Effect on Lattice Thermal Conductivity of Clathrates from First Principles. Phys. Rev. Lett. 2018, 120, 105901.

(24) Zhou, F.; Nielson, W.; Xia, Y.; Ozoliņ̌s, V. Lattice Anharmonicity and Thermal Conductivity from Compressive Sensing of First-Principles Calculations. Phys. Rev. Lett. 2014, 113, 185501.

(25) Monserrat, B.; Drummond, N. D.; Needs, R. J. Anharmonic Vibrational Properties in Periodic Systems: Energy, Electron-phonon Coupling, and Stress. Phys. Rev. B 2013, 87, 144302.

(26) Engel, E. A.; Monserrat, B.; Needs, R. J. Anharmonic Nuclear Motion and the Relative Stability of Hexagonal and Cubic ice. Phys. Rev. X 2015, 5, 021033.

(27) Prentice, J. C. A.; Needs, R. J. Using Forces to Accelerate First-principles Anharmonic Vibrational Calculations. Phys. Rev. Materials. 2017, 1, 023801.

(28) Souvatzis, P.; Eriksson, O.; Katsnelson, M. I.; Rudin, S. P. Entropy Driven Stabilization of Energetically Unstable Crystal Structures Explained from First Principles Theory. Phys. Rev. Lett. 2008, 100, 095901. 
(29) Errea, I.; Calandra, M.; Mauri, F. Anharmonic Free Energies and Phonon Dispersions from the Stochastic Self-Consistent Harmonic Approximation: Application to Platinum and Palladium Hydrides. Phys. Rev. B 2014, 89, 064302.

(30) Parlinski, K. Ab Initio Determination of Anharmonic Phonon Peaks. Phys. Rev. B 2018, 98, 054305 .

(31) Dovesi, R.; Erba, A.; Orlando, R.; Zicovich-Wilson, C. M.; Civalleri, B.; Maschio, L.; Rérat, M.; Casassa, S.; Baima, J.; Salustro, S. et al. Quantum-Mechanical Condensed Matter Simulations with CRYSTAL. Wiley Interdiscip. Rev. Comput. 2018, 8.

(32) Erba, A.; Maul, J.; Ferrabone, M.; Dovesi, R.; Rérat, M.; Carbonière, P. Anharmonic Vibrational States of Solids from DFT Calculations. Part II: Implementation of the VSCF and VCI Methods. J. Chem. Theory Comput. 2019, 15, 3766-3777.

(33) Erba, A.; Maul, J.; Ferrabone, M.; Carbonniére, P.; Rérat, M.; Dovesi, R. Anharmonic Vibrational States of Solids from DFT Calculations. Part I: Description of the Potential Energy Surface. J. Chem. Theory Comput. 2019, 15, 3755-3765.

(34) Carbonniére, P.; Erba, A.; Richter, F.; Dovesi, R.; Rérat, M. Calculation of Anharmonic IR and Raman Intensities for Periodic Systems from DFT Calculations: Implementation and Validation. J. Chem. Theory Comput. 2020, 16, 3343-3351.

(35) Maul, J.; Spoto, G.; Mino, L.; Erba, A. Elucidating the Structure and Dynamics of CO ad-layers on MgO Surfaces. Phys. Chem. Chem. Phys. 2019, 21, 26279-26283.

(36) Cherubini, M.; Monacelli, L.; Mauri, F. The Microscopic Origin of the Anomalous Isotopic Properties of Ice Relies on the Strong Quantum Anharmonic Regime of Atomic Vibration. J. Chem. Phys. 2021, 155, 184502.

(37) Bergren, M. S.; Rice, S. A. An Improved Analysis of the OH Stretching Region of the Vibrational Spectrum of Ice Ih. J. Chem. Phys. 1982, 77, 583-602. 
(38) Sceats, M. G.; Rice, S. A. The Intramolecular Potential of Water Molecules Engaged in Hydrogen Bonding from Analysis of the Overtone Spectrum of Ice I. J. Chem. Phys. 1979, 71, 973-982.

(39) Kapil, V.; Engel, E.; Rossi, M.; Ceriotti, M. Assessment of Approximate Methods for Anharmonic Free Energies. J. Chem. Theory Comput. 2019, 15, 5845-5857.

(40) Herrero, C. P.; Ramírez, R. Path-Integral Simulation of Ice VII: Pressure and Temperature Effects. Chem. Phys. 2015, 461, 125-136.

(41) Knuts, S.; Ojamäe, L.; Hermansson, K. An Ab Initio Study of the OH Stretching Frequencies in Ice II, Ice VIII, and Ice IX. J. Chem. Phys. 1993, 99, 2917-2928.

(42) Engel, E. A.; Monserrat, B.; Needs, R. J. Anharmonic Nuclear Motion and the Relative Stability of Hexagonal and Cubic ice. Phys. Rev. X 2015, 5, 021033.

(43) Burnham, C. J.; Reiter, G. F.; Mayers, J.; Abdul-Redah, T.; Reichert, H.; Dosch, H. On the Origin of the Redshift of the OH Stretch in Ice Ih: Evidence from the Momentum Distribution of the Protons and the Infrared Spectral Density. Phys. Chem. Chem. Phys. 2006, 8, 3966-3977.

(44) Senesi, R.; Flammini, D.; Kolesnikov, A. I.; Murray, E. D.; Galli, G.; Andreani, C. The Quantum Nature of the OH Stretching Mode in Ice and Water Probed by Neutron Scattering Experiments. J. Chem. Phys. 2013, 139, 074504.

(45) Becke, A. D. Density-Functional Exchange-Energy Approximation with Correct Asymptotic Behavior. Phys. Rev. A 1988, 38, 3098-3100.

(46) Lee, C.; Yang, W.; Parr, R. G. Development of the Colle-Salvetti Correlation-Energy Formula into a Functional of the Electron Density. Phys. Rev. B 1988, 37, 785-789.

(47) Krishnan, R.; Binkley, J. S.; Seeger, R.; Pople, J. A. Self-consistent Molecular Orbital 
Methods. XX. A Basis Set for Correlated Wave Functions. J. Chem. Phys. 1980, 72, $650-654$.

(48) Frisch, M. J.; Pople, J. A. Self-Consistent Molecular Orbital Methods 25. Supplementary Functions for Gaussian Basis Sets. J. Chem. Phys. 1984, 80, 3265.

(49) Pascale, F.; Zicovich-Wilson, C. M.; Gejo, F. L.; Civalleri, B.; Orlando, R.; Dovesi, R. The Calculation of the Vibrational Frequencies of Crystalline Compounds and its Implementation in the CRYSTAL Code. J. Comput. Chem. 2004, 25, 888-897.

(50) Zicovich-Wilson, C. M.; Pascale, F.; Roetti, C.; Saunders, V. R.; Orlando, R.; Dovesi, R. Calculation of the Vibration Frequencies of $\alpha$-quartz: The Effect of Hamiltonian and Basis Set. J. Comput. Chem. 2004, 25, 1873-1881.

(51) Maschio, L.; Kirtman, B.; Rérat, M.; Orlando, R.; Dovesi, R. Ab initio analytical Raman Intensities for Periodic Systems through a Coupled Perturbed Hartree-Fock/KohnSham Method in an Atomic Orbital Basis. II. Validation and Comparison with Experiments. J. Chem. Phys. 2013, 139, 164102.

(52) Maschio, L.; Kirtman, B.; Rérat, M.; Orlando, R.; Dovesi, R. Ab initio Analytical Raman Intensities for Periodic Systems through a Coupled Perturbed Hartree-Fock/KohnSham Method in an Atomic Orbital Basis. I. Theory. J. Chem. Phys. 2013, 139, 164101.

(53) Banks, P.; Song, Z.; Ruggiero, M. Assessing the Performance of Density Functional Theory Methods on the Prediction of Low-Frequency Vibrational Spectra. 2020,

(54) Erba, A.; Casassa, S.; Maschio, L.; Pisani, C. DFT and Local-MP2 Periodic Study of the Structure and Stability of Two Proton Ordered Polymorphs of Ice. J. Phys. Chem. B 2009, 113, 2347.

(55) Howe, R.; Whitworth, R. W. A Determination of the Crystal Structure of Ice XI. J. Chem. Phys. 1989, 4450. 
(56) Shigenari, T.; Abe, K. Vibrational Modes of Hydrogens in the Proton Ordered Phase XI of Ice: Raman Spectra Above $400 \mathrm{~cm}^{-1}$. J. Chem. Phys. 2012, 174504.

(57) Erba, A.; Casassa, S.; Dovesi, R.; Maschio, L.; Pisani, C. Periodic Density Functional Theory and Local-MP2 Study of the Librational Modes of Ice XI. J. Chem. Phys. 2009, 130,074505 .

(58) Banks, P. A.; Maul, J.; Mancini, M. T.; Whalley, A. C.; Erba, A.; Ruggiero, M. T. Thermoelasticity in organic semiconductors determined with terahertz spectroscopy and quantum quasi-harmonic simulations. J. Mater. Chem. C 2020, 8, 10917-10925.

(59) Hutereau, M.; Banks, P. A.; Slater, B.; Zeitler, J. A.; Bond, A. D.; Ruggiero, M. T. Resolving Anharmonic Lattice Dynamics in Molecular Crystals with X-Ray Diffraction and Terahertz Spectroscopy. Phys. Rev. Lett. 2020, 125, 103001.

(60) King, M. D.; Buchanan, W. D.; Korter, T. M. Investigating the Anharmonicity of Lattice Vibrations in Water-Containing Molecular Crystals through the Terahertz Spectroscopy ofl-Serine Monohydrate. J. Phys. Chem. A 2010, 114, 9570-9578. 